Artículo Original

Cinética de foto degradación de metomilo a través tecnologías de oxidación avanzadas

\title{
Metomyl photo-degradation kinetics through advanced oxidation technologies
}

\author{
Fátima Alonso $^{1}$, Danilo Fernández Ríos ${ }^{1} \&$ Alberto L. Capparelli² \\ ${ }^{1}$ Universidad Nacional de Asunción, Facultad de Ciencias Exactas y Naturales. San Lorenzo, Paraguay. fatilu777@hotmail.com; \\ dfernandez@facen.una.py \\ ${ }^{2}$ UNLP, CCT La Plata-CONICET, Facultad de Ciencias Exactas, Departamento de Química, Instituto de Investigaciones Fisicoquímicas \\ Teóricas y Aplicadas (INIFTA), La Plata, Argentina. alcappa2000@yahoo.com.ar
}

\begin{abstract}
Resumen: La fotodegradación de contaminantes mediante procesos de oxidación avanzada (POA) constituye un campo de investigación moderno en la química y la fisicoquímica ambiental. En este trabajo se presentan los progresos alcanzados en el área, ejemplificados mediante el desarrollo de un fotorreactor de diseño sencillo que aprovecha la luz solar para la degradación de insecticidas de uso frecuente en Paraguay, de potenciales aplicaciones en regiones rurales o aisladas. Como ejemplo de aplicación se seleccionó el insecticida metomilo, de amplio uso en la agricultura de Paraguay.
\end{abstract}

Palabras Clave: Fotocatálisis heterogénea, metomilo, $\mathrm{TiO}_{2}$, fotorreactor.

\begin{abstract}
The photodegradation of contaminants through advanced oxidation processes (POA) constitutes a modern research field in chemistry and environmental physical chemistry. This paper presents the advances made in said field, which are exemplified through the development of a photoreactor of simple design which takes advantage of sunlight tfor the degradation of frequently used insecticides in Paraguay, with potential applications in rural or isolated regions. As an example of application, the insecticide Methomyl was selected, which is widely used in agriculture in Paraguay.
\end{abstract}

KeyWords: Heterogeneous photocatalysis, methomyl, $\mathrm{Ti}_{2}$, photoreactor.

\section{Introducción}

El tratamiento de aguas contaminadas es un aspecto importante en la química, fisicoquímica y tecnologías ambientales asociadas(Friedmann et al., 2010). Como consecuencia del deterioro progresivo del ambiente, constantemente se desarrollan procedimientos ecocompatibles para impulsar tecnologías sostenibles y amigables con el ambiente.

Las aguas contaminadas pueden ser eficientemente tratadas en plantas biológicas convencionales (Malato et al., 2007). Sin embargo, existen contaminantes refractarios a los tratamientos biológicos y a los tratamientos normales de oxidación, o los procesos de su degradación son cinéticamente lentos.

Desde hace unos años, se han incorporado procedimientos basados en la radiación electromagnética para promover la fotodegradación en el tratamiento de contaminantes refractarios(Malato Rodríguez et al., 2001). Tales procedimientos se conocen en la literatura como Procesos de Oxidación Avanzados(POA) (Sievers, 2011), algunos

Recibido: 23/07/2019 de los cuales han alcanzado un nivel de aplicación industrial en países desarrollados y en vías de desarrollo. Entre estos métodos se incluyen los procedimientos homogéneos VUV (VacuumUltraviolet o ultravioleta del vacío), $\mathrm{UV} / \mathrm{H}_{2} \mathrm{O}_{2}, \mathrm{UV} / \mathrm{O}_{3}, \mathrm{UV} /$ $\mathrm{H}_{2} \mathrm{O}_{2} / \mathrm{O}_{3}, \mathrm{UV} / \mathrm{S}_{2} \mathrm{O}_{8}=$, Fenton $\left(\mathrm{Fe}^{+3} / \mathrm{Fe}^{+2}+\mathrm{H}_{2} \mathrm{O}_{2}\right.$, foto Fenton, y heterogéneos que emplean $\mathrm{TiO}_{2}$ como fotocatalizador entre los más frecuentes.

Por ejemplo, la siguiente oxidación:

$$
\text { ( }
$$

(Reacción 1)

es una reacción termodinámicamente espontánea pero cinéticamente lenta (Reacción 1). En consecuencia, es un compuesto refractario a los procesos de oxidación, siendo un excelente sustrato para la aplicación de los procesos de oxidación avanzada (POA).

Aceptado: 03/10/2019 
Estos procesos, de base fisicoquímica, producen transformaciones irreversibles en la estructura química del contaminante a ser eliminado, generando especies radicales normalmente inestables que reaccionan formando otros tipos de radicales hasta generar especies estables, o bien hasta la mineralización completa de la sustancia. Los cambios estructurales producidos y la generación de intermediarios oxidan la materia orgánica. Entre estos intermediarios juega un papel central el radical hidroxilo $(\mathrm{HO} \bullet)$, que es un oxidante inespecífico y muy eficiente para iniciar el proceso de oxidación de la materia orgánica. La baja especificidad y alta reactividad son la base de su uso extendido para eliminar contaminantes refractarios en aguas. Esta especie posee propiedades adecuadas para atacar virtualmente a todos los compuestos orgánicos y reaccionar $10^{6}-10^{12}$ veces más rápido que oxidantes alternativos como el $\mathrm{O}_{3}$.

Tabla 1. Potencial de oxidación de distintas especies reactivas de interés ambiental.

\begin{tabular}{l|c}
\hline Oxidante & Potencial de oxidación (V) a $\mathbf{2 5}^{\circ} \mathbf{C}$ \\
\hline Flúor & 3,03 \\
\hline $\mathrm{HO}^{\circ}$ & 2,80 \\
$\mathrm{O}\left({ }^{1} \mathrm{D}\right)$ & 2,42 \\
$\mathrm{O}_{3}$ & 2,07 \\
$\mathrm{H}_{2} \mathrm{O}_{2}$ & 1,77 \\
$\mathrm{H}_{2} \mathrm{O}^{\cdot}$ & 1,78 \\
$\mathrm{MnO}_{4}^{-}$ & 1,68 \\
$\mathrm{ClO}_{2}$ & 1,50 \\
$\mathrm{Cl}_{2}$ & 1,36 \\
$\mathrm{O}_{2}$ & 1,23 \\
\hline
\end{tabular}

Las capacidades de oxidación de distintos tipos de intermediarios se presentan en la Tabla 1, ordenadas por su potencial de oxidación. A excepción del flúor, el radical HO posee un potencial redox elevado(García Einschlag, 2001; López, 2008).

Entre los objetivos de los POA se encuadra el desarrollo de métodos fisicoquímicos de bajo costo para el tratamiento de efluentes, con la eventual posibilidad de emplear radiación solar y así facilitar la mineralización de los contaminantes a anhídrido carbónico $\left(\mathrm{CO}_{2}\right)$, agua e iones inorgánicos como el ion cloruro $\left(\mathrm{Cl}^{-}\right)$(Strathmann \& Stone, 2001).

El empleo de métodos alternativos y procedimientos fotoquímicos en general puede constituir una vía adecuada para la eliminación o reducción de estos contaminantes primarios y secundarios a niveles de toxicidad tolerables, o transformarlos en productos susceptibles a los tratamientos biológicos convencionales.

La degradación de contaminantes por medio dela irradiación de soluciones que contienen dióxido de titanio disperso o soportado sobre materiales inertes es un campo de rápido crecimiento en la investigación básica y aplicada. El desarrollo de este proceso a fin de lograrla mineralización completa de contaminantes orgánicos ha sido ampliamente probado para una gran variedad de productos químicos (Legrini et al., 1993).

Los radicales $\mathrm{HO}^{\circ}$ pueden experimentar distintos procesos con los constituyentes presentes en el medio. Éstos pueden agruparse en general de la siguiente manera:

Abstraer $\mathrm{H}$ de compuestos alifáticos(Reacción 2), generando radicales susceptibles de reaccionar con el $\mathrm{O}_{2}$ presente para formar radicales peróxidos de reactividad variable que pueden conducir a su mineralización completa (formar $\mathrm{CO}_{2} \mathrm{y}$ ácidos inorgánicos, dependiendo de si existen sustituyentes halogenados, etc.) (Reacción 3):

$$
\begin{gathered}
\mathrm{HO}^{\bullet}+\mathrm{RH} \rightarrow \mathrm{H}_{2} \mathrm{O}+\mathrm{R}^{\bullet}(\text { Reacción 2) } \\
\mathrm{R}^{\bullet}+\mathrm{O}_{2} \rightarrow \mathrm{RO}_{2}^{\bullet} \rightarrow \rightarrow \rightarrow \mathrm{CO}_{2}+\mathrm{H}_{2} \mathrm{O}(\text { Reacción 3) }
\end{gathered}
$$

Adicionarse a dobles enlaces en alquenos, por ejemplo, o compuestos aromáticos, generando nuevos radicales que pueden conducir a productos (mineralizarse completamente o bien formar subproductos de toxicidad variable) (Reacción 4).

$$
\mathrm{HO}^{\bullet}+\mathrm{Ar} \rightarrow \mathrm{HOAr}^{\bullet} \rightarrow \text { Productos (Reacción 4) }
$$

Sufrir reacciones de transferencia de electrones, generando radicales cationes, que en agua son inestables (Reacción 5), y en consecuencia experi- 
mentar distintas reacciones para generar productos (Reacción 6).

$$
\begin{gathered}
H O^{\bullet}+R X \rightarrow H O^{-}+R X^{+}(\text {Reacción 5) } \\
R X^{+} \rightarrow \rightarrow \text { Productos }(\text { Reacción } 6)
\end{gathered}
$$

Las principales ventajas de estos métodos consisten en que existe transformación de las sustancias contaminantes y son adecuados para procesos de pretratamientos a los métodos convencionales cuando los contaminantes son refractarios a éstos(es importante conocer si los subproductos de oxidación serán tóxicos previamente al paso convencional). Además, es posible alcanzar la mineralización completa (destrucción) del contaminante, mientras que las tecnologías convencionales que no emplean especies muy fuertemente oxidantes no alcanzan a oxidar completamente la materia orgánica. Otra ventaja es que no se generan barros que requieran de un proceso de tratamiento y/o disposición, y sirven para tratar contaminantes en bajas concentraciones de manera que la radiación penetre en el reactor fotoquímico (o fotorreactor).

Una de las principales desventajas es el consumo de sustancias de alto costo y/o energía eléctrica. En algunos casos, consumen mucha menos energía que otros métodos (por ejemplo, la incineración). Además, pueden originarse subproductos de mayor toxicidad que las sustancias a degradar. Por este motivo, se debe controlar el contenido de carbono orgánico total junto con el contenido de carbono orgánico específico de la sustancia estudiada.

\section{Fotocatálisis heterogénea}

Los procesos fotocatalíticos son aquellos en los cuales la velocidad de reacción se incrementa como respuesta a la excitación luminosa de un sólido que permanece sin modificaciones, o mediante la excitación de la molécula adsorbida sobre el sólido sin que tampoco experimente cambios.

En los métodos basados en el empleo de semiconductores como fotocatalizadores, la absorción de radiación promueve electrones a la banda de conducción, dejando un hueco en la banda de valencia. En estos procesos la formación de radicales
$\mathrm{HO}^{\bullet}$ es maximizada por la adición de oxidantes como $\mathrm{H}_{2} \mathrm{O}_{2} \mathrm{u} \mathrm{O}_{3}$.

Un fotocalizador como semiconductor se caracteriza porque existe una diferencia de energía entre la banda de valencia (bv) y la de conducción (bc). Cuando un semiconductor es irradiado por luz cuya energía es mayor que la diferencia de energía entre ambas bandas, los electrones son promovidos desde la banda de valencia hasta la de conducción, donde estos electrones ( $\left.\mathrm{e}^{-}\right)$presentarán la capacidad de reducir a las moléculas adsorbidas sobre la superficie; mientras que en la banda de valencia se genera un hueco positivo $\left(\mathrm{h}^{+}\right)$que promoverá la oxidación de las moléculas sobre el fotocatalizador.

Uno de los fotocatalizadores más estudiados es el $\mathrm{TiO}_{2}$, que absorbe luz de $\lambda$ menores a $380 \mathrm{~nm}$. En este proceso se genera un par electrón $\left(\mathrm{e}^{-}\right) /$hueco $\left(\mathrm{h}^{+}\right)$según la reacción $\mathrm{TiO}_{2}+\mathrm{h} \nu \rightarrow \mathrm{e}^{-}+\mathrm{h}^{+}$. Este par $\mathrm{e}^{-} / \mathrm{h}^{+}$puede recombinarse rápidamente o si alcanzan la interfase del semiconductor donde pueden existir iones $\mathrm{OH}^{-}$, moléculas de agua adsorbidas, etc.; reaccionar formando, por ejemplo, el radical $\mathrm{HO}^{\bullet}$ sobre la superficie del fotocatalizador, según la reacción $\mathrm{h}^{+}+\mathrm{OH}^{-} \rightarrow \mathrm{HO}^{\circ}(\mathrm{ads})$. Este radical inicia una serie de procesos de oxidación de los contaminantes. En la Figura 1 y en las reacciones 7-14 se esquematizan las etapas principales del proceso.

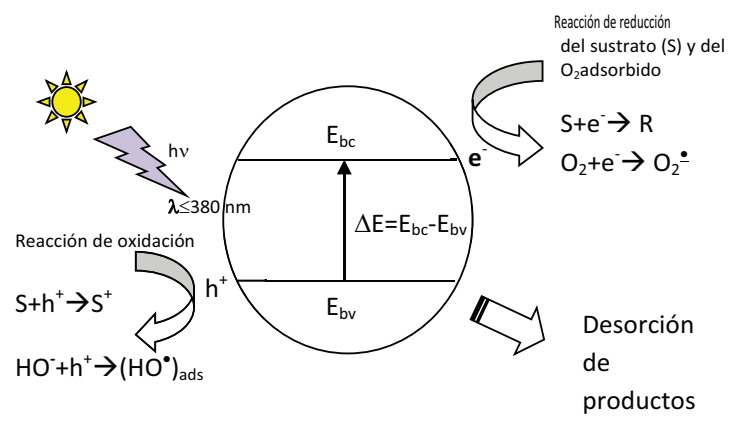

Figura 1. Esquema de los procesos sobre una partícula de fotocatalizador. Adaptado a partir de Química y Fisicoquímica Ambiental (Etchegoyen et al., 2020).

Los electrones $\mathrm{e}^{-}$se consumen por la presencia de $\mathrm{O}_{2}$, generando el anión superóxido. Todas estas especies participan de las reacciones de oxidación de contaminantes. Respecto de los casos anteriores, lo que ha variado es el mecanismo 
de generación de estos radicales. Las ventajas de este tipo de procedimiento radican en que un gran número de sustancias pueden ser mineralizadas completamente con una fuerte influencia del área superficial, el costo del fotocatalizador es relativamente bajo, es potencialmente aplicable en regiones UV propias del espectro solar, y existe la posibilidad de dopar el material o de construir matrices sólidas con dióxido de titanio. Las mayores desventajas son su bajo rendimiento cuántico, y que en sistemas donde el fotocalizador está en suspensión una fracción importante de la radiación es dispersada. Por este motivo, la investigación está orientada a desarrollar fotocalizadores fijos sobre distintos tipos de soportes. En estos procesos el papel del oxígeno es fundamental para que la mineralización sea efectiva.

El mecanismo de reacción general es el siguiente:

$$
\begin{gathered}
\mathrm{TiO}_{2}+h v \rightarrow\left(\mathrm{TiO}_{2}\right) \rightarrow \mathrm{TiO}_{2}\left(e_{b c}^{-}+h_{b v}^{+}\right)(\text {Reacción } 7) \\
\mathrm{HO}^{-}+h_{b v}^{+} \rightarrow \mathrm{HO}^{\bullet} \quad(\text { Reacción } 8) \\
\mathrm{O}_{2}+e_{b c}^{-} \rightarrow \mathrm{O}_{2}^{-} \text {(Reacción 9) } \\
\mathrm{O}_{2}^{-}+\mathrm{H}^{+} \rightarrow \mathrm{HO}_{2}^{\bullet} \text { (Reacción 10) } \\
\mathrm{HO}_{2}^{-}+\mathrm{HO}_{2}^{\bullet} \rightarrow \mathrm{H}_{2} \mathrm{O}_{2}+\mathrm{O}_{2}(\text { Reacción 11) } \\
\mathrm{HO}_{2}^{\bullet}+\mathrm{H}_{2} \mathrm{O} \rightarrow \mathrm{H}_{2} \mathrm{O}_{2}+\mathrm{HO}^{\bullet} \text { (Reacción 12) } \\
\mathrm{H}_{2} \mathrm{O}_{2}+\mathrm{O}_{2} \rightarrow \mathrm{HO}^{-}+\mathrm{HO}^{\bullet}+\mathrm{O}_{2} \text { (Reacción 13) } \\
\mathrm{H}_{2} \mathrm{O}_{2}+e_{b c}^{-} \rightarrow \mathrm{HO}^{-}+\mathrm{HO}^{\bullet} \text { (Reacción 14) }
\end{gathered}
$$

A partir de estas reacciones se inicia una serie de procesos en los que se generan radicales libres e intermediarios de reacción (Reacciones 7 a 14). La aplicabilidad del $\mathrm{TiO}_{2}$ en este campo ha sido demostrada no sólo en suspensión, sino además en diferentes tipos de soportes, tales como vidrios o plásticos PET mediante impregnación (Meichtry et al., 2007).

En la presente revisión se describe la aplicación del POA basado en la fotocatálisis con $\mathrm{TiO}_{2}$ para la degradación de insecticidas refractarios con alta solubilidad en agua y de uso intensivo en el país.

\section{Materiales y métodos}

Se utilizaron los siguientes reactivos: metomilo con $92,8 \%$ de pureza (materia prima) y Dióxido de Titanio $\left(\mathrm{TiO}_{2}\right)$ marca Kronos con título de 99,1\%. El agua ultrapura con conductividad de $0,05 \mu \mathrm{S}$ y $\mathrm{COD}_{\text {max: }} 0,001 \mathrm{mg} . \mathrm{L}^{-1}$ fue obtenida a partir de un desionizador de agua de marca Thermo Scientific modelo Barnstead Gen Pure ${ }^{\mathrm{TM}}$.

Los soportes utilizados para impregnación con $\mathrm{TiO}_{2}$ fueron perlas de porcelana de $6 \mathrm{~mm}$ de diámetro y $1,13 \mathrm{~cm}^{2}$ de superficie, y botellas PET de un litro de capacidad.

Para el monitoreo de la concentración de los contaminantes en las muestras de degradación por fotocatálisis pueden seguir aplicándose técnicas analíticas modernas, como la cromatografía líquida de alta resolución con detector ultravioleta con arreglo de diodos (HPLC/UV-DAD); siendo adecuado para este tipo de análisis el método cromatográfico isocrático, en el cual se puede emplear como fase móvil una mezcla de $80 \%$ de agua ultra pura y $20 \%$ de acetonitrilo. En función de las características del contaminante y de sus propiedades fisicoquímicas se debe evaluar la longitud de onda de seguimiento yel tipo de columna de fase reversa (Por ejemplo, Merck RP-8 $(5 \mu \mathrm{m})$ de relleno octilsilano $250 \mathrm{~mm}$ $\times 4,6 \mathrm{~mm} \times 4 \mu \mathrm{m}$ de tamaño de partícula).

\section{Construcción de fotorreactor solar}

El diseño de fotorreactores sencillos con superficie semiparabólica reflectante de la luz solar incidente es viable, y para su construcción se requiere evaluar distintos tipos de materiales. En un estudio previo realizado en este laboratorio(Alonso Espínola, 2018),uno de los dispositivos estuvo constituido por un balón de vidrio de cuatro litros de capacidad provisto por una tapa esmerilada móvil con varias aberturas que fueron utilizadas para introducir un termómetro, el agitador manual y permitir la entrada de oxígeno. El balón se colocó sobre un soporte metálico. Para incrementar la cantidad de radiación incidente en el medio de reacción, se utilizó como superficie reflectora una plancha de aluminio pulido de $30 \mathrm{~cm}$ x $40 \mathrm{~cm}$ ubicado alrededor del reactor esférico. La temperatura de trabajo fue supervisada 
durante todo el proceso, alcanzando temperaturas de hasta $55^{\circ} \mathrm{C}$ en los reactores debido a la luz solar, así como la forma de agregado del fotocatalizador. Otro modelo de fotorreactor utilizado fue una superficie semiparabólica sencilla cubierta con hoja de aluminio y bloques ubicados al dorso que permitieron la variación del ángulo. El recipiente que contenía la muestra (botella PET de 1litro) se colocó en el centro del reactor(Alonso Espínola, 2018; Meichtry et al., 2007).

\section{Procedimiento de impregnación de soportes con $\mathrm{TiO} 2$}

E1 $\mathrm{TiO}_{2}$ utilizado con fotocatalizador puede ser incorporado por medio de la impregnación de perlas de vidrio(Serpone et al., 1986), que pueden prepararse siguiendo los protocolos descritos en la literatura (Litter, 2005; Piperata et al., 2004). Como ejemplo, para la impregnación de las perlas de porcelana se deja durante dos horas una cantidad específica en contacto con una solución de ácido nítrico 0,1 M. Luego se lava y seca a $100^{\circ} \mathrm{C}$. El $\mathrm{TiO}_{2}$ para impregnar se trata previamente de la siguiente manera: se prepara una suspensión al 1\% (p/v) cuyo $\mathrm{pH}$ se ajusta a 2,5 con ácido perclórico $\left(\mathrm{HClO}_{4}\right)$, y se sonica durante media hora. Las perlas previamente tratadas se colocan en contacto con esta suspensión en una cápsula de porcelana hasta su completa inmersión y se llevan a evaporación lenta hasta sequedad en una plancha calentadora. El proceso se repite hasta obtener una segunda capa. Finalmente se fija el $\mathrm{TiO}_{2}$ a una temperatura de $450^{\circ} \mathrm{C}$ por dos horas. Se enfría, lava con agua destilada y se deja secar en estufa a 120 ${ }^{\circ} \mathrm{C}$ durante 20 minutos.

Otra técnica se basa en el uso de botellas PET limpias y secas a temperatura ambiente. La impregnación se alcanza empleando una suspensión de $\mathrm{TiO}_{2}$ al $1 \%(\mathrm{p} / \mathrm{v})$ previamente ajustado el $\mathrm{pH}$ a 2,5 con gotas de ácido nítrico concentrado; se agita suavemente a fin de obtener una capa homogénea sobre la pared. Se deja secar a temperatura ambiente por 24 horas. El sistema se lava y se agita vigorosamente en forma manual.

\section{Fotocatálisis bajo irradiación solar}

Se realizaron ensayos para medir la adsorción de metomilo $\left(\mathrm{C}_{5} \mathrm{H}_{10} \mathrm{~N}_{2} \mathrm{O}_{2} \mathrm{~S}\right)$ en una concentración inicial de $10 \mathrm{mg} . \mathrm{L}^{-1}$ en agua destilada sobre $200 \mathrm{mg} . \mathrm{L}^{-1}$ de fotocatalizador $\mathrm{TiO}_{2}$ impregnado, en los cuales se dejó en contacto la solución con el soporte impregnado a oscuras durante cierto tiempo; y ensayos de fotólisis mediante exposición directa de la solución a la luz solar con el fin de determinar si el principio activo es fotodegradable con luz y en ausencia de fotocatalizador como blanco.

Como se conoce para otros contaminantes refractarios (García Einschlag, 2001; López, 2008), el metomilo no experimenta fotodegradación por efecto de la fotólisis en ausencia del fotocatalizador. Asimismo, no se produce adsorción del pesticida sobre el fotocatalizador.

Las reacciones con luz solar directa se realizaron en días completamente soleados. Se colectaron muestras a diferentes tiempos de exposición, tras ser filtradas con filtro de $0,45 \mu \mathrm{m}$ se analizaron mediante HPLC-DAD. Se realizaron además lecturas de carbono orgánico total para analizar el nivel de mineralización del compuesto tras la fotodegradación.

\section{Resultados y discusión}

\section{Cinética de degradación de metomilo mediante fotocatálisis heterogénea: $\mathrm{TiO}_{2}$ impregnado en soportes}

Los resultados de la comparación dela fotodegradación heterogénea con impregnación sobre perlas

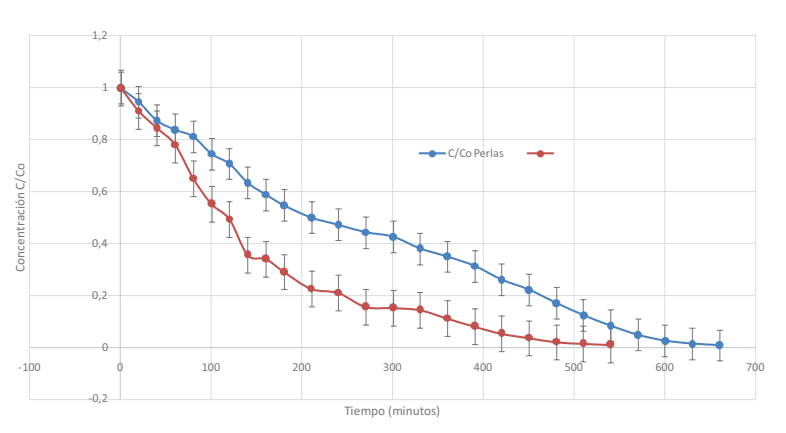

Figura 2. Perfil de degradación del metomilo mediante fotocatálisis heterogénea con soporte botellas PET y perlas $\left(\mathrm{TiO}_{2}\right.$ $=200 \mathrm{mg} . \mathrm{L}^{-1} \mathrm{y}$ radiación solar). 
y sobre superficies $\mathrm{PETconTiO}_{2}$ se presentan en la Figura 2, donde se observa un decaimiento de la concentración de metomilo en función del tiempo de exposición de las soluciones a la radiación solar. En promedio, alrededor del 98\% del metomilo se degradó al tiempo final de detección por HPLC en ambos tipos de tratamiento. Se observó comportamiento similar para ambos casos, aunque en el caso de las botellas se observó un porcentaje de degradación mayor en menores tiempos de exposición solar en comparación al obtenido con las perlas.

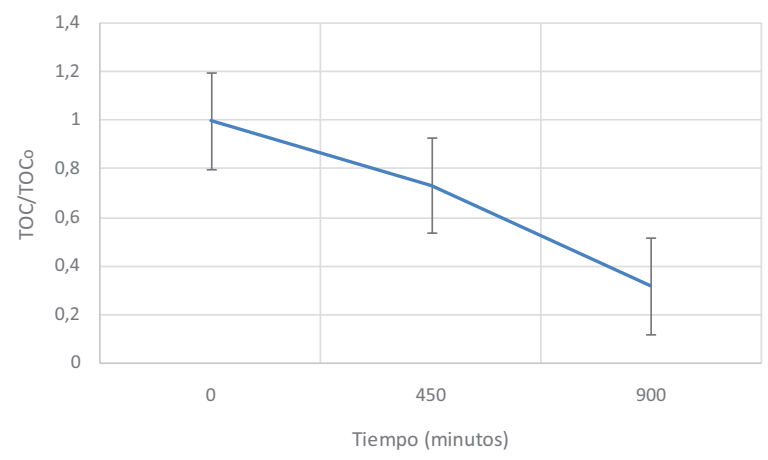

Figura 3. Perfil de mineralización del metomilo mediante fotocatálisis heterogénea.

\section{Perfil de mineralización y resultados experi- mentales}

En la Figura 3se muestra el perfil de mineralización del metomilo en función del tiempo de iluminación. Estas medidas se realizaron evaluando el contenido orgánico total (TOC por sus siglas en inglés).

La desaparición de materia orgánica se siguió evaluando el TOC de las soluciones irradiadas. Se observó una disminución del TOC del 68\%después de 900 minutos de exposición a la luz solar.

Del ajuste lineal del $\ln (\mathrm{C} / \mathrm{Co})$ en función del

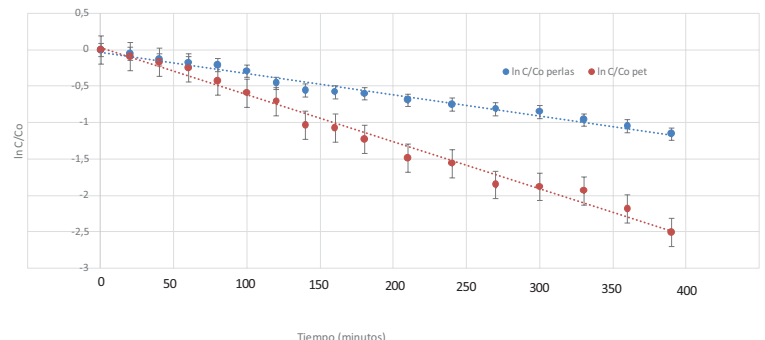

Figura 4. Comportamiento cinético de seudoprimer orden de soportes perlas versus PET.

tiempo (Figura 4) se obtienen los parámetros cinéticos de la reacción. Esto demuestra que la degradación del metomilo en agua mediante fotocatálisis heterogénea con $\mathrm{TiO}_{2}$ presenta un comportamiento cinético de seudoprimer orden.

De este tipo de análisis pueden obtenerse los tiempos medios de degradación. En el caso de perlas impregnadas el tiempo medio es de120 minutos, mientras que en botellas PET impregnadas es de 88 minutos.

Esta diferencia en los tiempos de degradación a favor de la segunda técnica puede asociarse a la mayor superficie de contacto entre la solución y el material impregnado. Además, en el reactor se logró mayor aprovechamiento solar con la superficie reflectora al registrarse temperaturas de hasta $55^{\circ} \mathrm{C}$ en el blanco en comparación con el reactor de vidrio, en el cual se registraron máximas de $47^{\circ} \mathrm{C}$ en el mismo día.

Otros autores (Juang \& Chen, 2014; Tomašević et al., 2010) también observaron una cinética de seudoprimer orden para la degradación del metomilo en disoluciones acuosas por fotocatálisis con $\mathrm{TiO}_{2}$ bajo irradiación UV con variación delas concentraciones de partida del plaguicida y de $\mathrm{TiO}_{2}$ en suspensión. En la Tabla 2 se indican algunos resultados comparativos reportados en la literatura.

\begin{tabular}{|c|c|c|c|c|c|}
\hline Medio & $\begin{array}{l}\text { Concentraciones } \\
\left(\mathrm{mg} \cdot \mathrm{L}^{-1}\right)\end{array}$ & $\begin{array}{l}\text { Constante de degradación } \\
\qquad\left(\mathrm{min}^{-1}\right)\end{array}$ & $\begin{array}{l}\text { Tiempo de vida media } \\
\text { (min) }\end{array}$ & pH & Referencias \\
\hline $\begin{array}{c}\text { Agua } \\
\text { destilada }\end{array}$ & $\begin{array}{l}\text { metomilo: } 10 \\
\mathrm{TiO}_{2}: 750\end{array}$ & 0,015 & 46 & 6 & (Juang \& Chen, 2014) \\
\hline $\begin{array}{l}\text { Agua } \\
\text { destilada }\end{array}$ & $\begin{array}{l}\text { metomilo: } 16,2 \\
\mathrm{TiO}_{2}: 200\end{array}$ & 0,0022 & 315 & 6 & (Tomašević et al., 2010) \\
\hline
\end{tabular}

Tabla 2. Estudios realizados con metomilo en solución acuosa, a diferentes concentraciones de plaguicida y $\mathrm{TiO}_{2}$. 


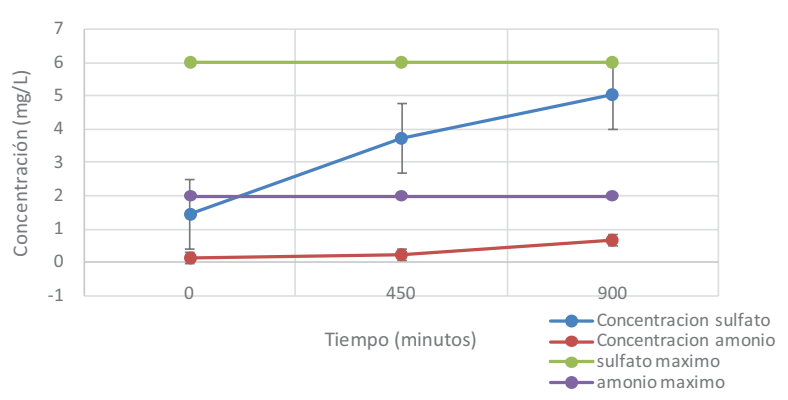

Figura 5. Iones inorgánicos $\left(\mathrm{NH}_{4}^{+}\right),\left(\mathrm{SO}_{4}^{-2}\right)$ procedentes de la degradación del metomilo. Concentración inicial de metomilo de $10 \mathrm{mg} \cdot \mathrm{L}^{-1},\left(\mathrm{SO}_{4}^{-2}\right)$ máximo: $6 \mathrm{mg} \cdot \mathrm{L}^{-1},\left(\mathrm{NH}_{4}^{+}\right)$máximo: 2 mg. $\mathrm{L}^{-1}$.

\section{Caracterización e identificación de subproductos de reacción}

La evolución de las concentraciones de iones inorgánicos en disolución — nitrógeno en forma de amonio y azufre en forma de sulfato- liberados a partir de la molécula original de plaguicida durante el proceso fotocatalítico se presentan en la Figura 5.

Se observó un incremento en las concentraciones del ion sulfato y del amonio en el transcurso de la degradación. Partiendo de una concentración de metomilo de $10 \mathrm{mg} . \mathrm{L}^{-1}$, el máximo de amonio por estequiometria es de $2 \mathrm{mg}$. $\mathrm{L}^{-1}$, y el de sulfato $6 \mathrm{mg}$. $\mathrm{L}^{-1}$.Los resultados experimentales obtenidos fueron de $0,687 \mathrm{mg} . \mathrm{L}^{-1}$, correspondiente al $31,2 \%$ del máximo para el amonio; y 5,048mg.L${ }^{1}$,correspondiente al $84,1 \%$ del máximo del sulfato del valor que debe resultar de la mineralización completa del contaminante.

Se puede suponer la mineralización del metomilo tras el tratamiento, aunque en ninguno de los casos se ha logrado el100\% de mineralización pasados los 900 minutos de degradación. En el caso del anión $\mathrm{SO}_{4}{ }^{-2}$, la posible adsorción de esta especie puede determinar una menor concentración como fracción soluble, tal como ha sido propuesto en la literatura (Tamimi et al., 2006).

\section{Conclusiones}

En promedio, alrededor del 98\% se degradó al tiempo final de detección por HPLC con el tratamiento. Así, cuando el metomilo (seguido por HPLC) se reduce al $98 \%$ de la concentración inicial, las medidas de TOC indican que la carga orgánica es del orden del $50 \%$ de la inicial. Esto es un indicador de la presencia de intermediarios orgánicos como subproductos de la degradación del contaminante. A los 900 minutos de exposición de la solución a la radiación solar incidente, el contenido de la carga orgánica total continúa siendo alto, del orden del $32 \%$ de la inicial. Consecuentemente, la eliminación completa del contenido orgánico debe ser mayor a los 900 minutos de irradiación.

Durante el proceso se forman especies inorgánicas. En particular se detectó la presencia de amonio y de sulfatos en la solución irradiada.

La aplicación de técnicas de oxidación avanzadas constituye una herramienta de alto potencial para la degradación de contaminantes refractarios a las técnicas convencionales aprovechando la radiación solar.

En el ámbito de influencia de la UNA, hemos contribuido a desarrollar una fotorreactor sencillo para el tratamiento fotocatalítico de aguas empleando la luz solar y un procedimiento foto-Fenton. Por sus características, fue el primero en ser empleado en el país.

La aplicabilidad de estos procesos a escala piloto o adecuada para el tratamiento de volúmenes apreciables de aguas requiere del desarrollo del diseño y construcción de fuentes de iluminación y de fotoreactores más adecuados, así el desarrollo de nuevos fotocatalizadores o sistemas de óxidoreducción más eficientes. La investigación básica es imprescindible para alcanzar estos objetivos.

\section{Agradecimientos}

Los autores desean agradecer a la Facultad de Ciencias Exactas y Naturales de la Universidad Nacional de Asunción y al Consejo Nacional de Ciencia y Tecnología de Paraguay (CONACYT) por su apoyo financiero, y a Nidia Benítez por la crítica constructiva del manuscrito.

\section{Literatura citada}

Alonso Espínola, F. L. (2018). Cinética de 
fotodegradación de metomilo empleando tecnologías de oxidación avanzadas. Tesis de Postgrado. San Lorenzo: Universidad Nacional de Asunción.

Etchegoyen, M. A., Marino, D. J. G., \& Capparelli, A. L. (2020). Tópicos de Química y Fisicoquímica Ambiental. Agua, atmósfera y suelo, transferencia entre compartimientos y transformaciones. Editorial de la Universidad Nacional de La Plata (EDULP). http://sedici.unlp.edu.ar/handle/10915/95979

Friedmann, D., Mendive, C., \& Bahnemann, D. (2010). TiO2 for water treatment: Parameters affecting the kinetics and mechanisms of photocatalysis. Applied Catalysis B: Environmental, 99(3-4): 398-406. https:// doi.org/10.1016/j.apcatb.2010.05.014

García Einschlag, F. S. (2001). Fotodegradación de compuestos nitroaromáticos mediante la técnica UV/H2O2. Facultad de Ciencias Exactas.

Juang, R.-S., \& Chen, C.-H. (2014). Comparative study on photocatalytic degradation of methomyl and parathion over UV-irradiated $\mathrm{TiO} 2$ particles in aqueous solutions. Journal of the Taiwan Institute of Chemical Engineers, 45(3): 989-995. https://doi. org/10.1016/j.jtice.2013.09.025

Legrini, O., Oliveros, E., \& Braun, A. M. (1993). Photochemical processes for water treatment. Chemical Reviews, 93(2): 671-698. https://doi.org/10.1021/cr00018a003

Litter, M. I. (2005). Introduction to Photochemical Advanced Oxidation Processes for Water Treatment. In Environmental Photochemistry Part II (pp. 325-366). Springer-Verlag. https://doi.org/10.1007/ b138188

López, J. L. (2008). Propiedades químicas fotoquímicas y ecotoxicológicas del ácido 4-cloro-3, 5-dinitrobenzoico en solución acuosa. Facultad de Ciencias Exactas.

Malato Rodríguez, S., Maldonado Rubio, I., \& Blanco Gálvez, J. (2001). Descontami- nación de aguas de lavado de envases de plaguicidas mediante fotocatálisis solar. Ministerio de Ciencia y Tecnologia. Centro de Investigaciones Energeticas Medioambientales y Tecnologicas.

Malato, S., Blanco, J., Alarcón, D. C., Maldonado, M. I., Fernández-Ibáñez, P., \& Gernjak, W. (2007). Photocatalytic decontamination and disinfection of water with solar collectors. Catalysis Today, 122(1-2): 137-149. https://doi.org/10.1016/j.cattod.2007.01.034

Meichtry, J. M., Lin, H. J., de la Fuente, L., Levy, I. K., Gautier, E. A., Blesa, M. A., \& Litter, M. I. (2007). Low-Cost TiO[sub 2] Photocatalytic Technology for Water Potabilization in Plastic Bottles For Isolated Regions. Photocatalyst Fixation. Journal of Solar Energy Engineering, 129(1): 119. https://doi.org/10.1115/1.2391317

Piperata, G., Meichtry, J. M., \& Litter, M. I. (2004). Photocatalytic reactions over $\mathrm{TiO} 2$ supported on porcelain spheres. In Surface and Colloid Science (Vol. 128, pp. 303-308). Springer Berlin Heidelberg. https://doi.org/10.1007/b97123

Serpone, N., Borgarello, E., Harris, R., Cahill, P., Borgarello, M., \& Pelizzetti, E. (1986). Photocatalysis over TiO2 supported on a glass substrate. Solar Energy Materials, 14(2): 121-127. https://doi. org/10.1016/0165-1633(86)90070-5

Sievers, M. (2011). Advanced Oxidation Processes. In Treatise on Water Science (pp. 377408). Elsevier. https://doi.org/10.1016/ B978-0-444-53199-5.00093-2

Strathmann, T. J., \& Stone, A. T. (2001). Reduction of the Carbamate Pesticides Oxamyl and Methomyl by Dissolved Fe II and $\mathrm{Cu}$ I. Environmental Science \& Technology, 35(12): 2461-2469. https://doi. org/10.1021/es001824j

Tamimi, M., Qourzal, S., Assabbane, A., Chovelon, J.-M., Ferronato, C., \& Ait-Ichou, Y. (2006). Photocatalytic 
degradation of pesticide methomyl: Tomašević, A., Kiss, E., Petrović, S., \& Midetermination of the reaction pathway and identification of intermediate products. Photochemical \& Photobiological Sciences, 5(5): 477. https://doi. org/10.1039/b517105a jin, D. (2010). Study on the photocatalytic degradation of insecticide methomyl in water. Desalination, 262(1-3): 228-234. https://doi.org/10.1016/j. desal.2010.06.019 